\title{
Contraindications to immunotherapy: a global approach
}

\author{
C. Pitsios ${ }^{1 *}$ D , M. Tsoumani ${ }^{2}$, M. B. Bilò ${ }^{3}$, G. J. Sturm ${ }^{4,5}$, P. Rodríguez del Río ${ }^{6}$, R. Gawlik, F. Ruëff ${ }^{8}$, \\ G. Paraskevopoulos ${ }^{9}$, E. Valovirta ${ }^{10}$, O. Pfaar ${ }^{11,12}$, M. A. Calderón ${ }^{13}$ and P. Demoly ${ }^{14,15}$
}

\begin{abstract}
Background: Recommendations on contraindications to allergen immunotherapy (AIT) have been independently developed by National and International Societies/Academies. AlT contraindications are mainly based on case reports, case-series, or experts' opinion, while evidence-based information is limited. The aim of the present review was to describe existing guidelines on contraindications to AIT and to highlight differences between them.

Main body: An extended review of the literature regarding contraindications to AIT for respiratory allergy and venom hypersensitivity was performed. Furthermore, Societies and Academies registered in the World Allergy Organization and EAACl databases, were asked for additional information. Only AIT guidelines published under official auspicies were included. A large heterogeneity among the various recommendations on contraindications was registered. Common contraindications to most of the guidelines were: lack of adherence, pregnancy before the start of AIT, the use of beta-blockers, certain age groups, uncontrolled asthma, autoimmune diseases and malignancies.

Conclusion: As new data arise, revisions might soon be needed allowing AIT in the cases of patients treated with ACE inhibitors and beta-blockers, in elderly patients and in patients with concomitant autoimmune diseases and neoplasias in remission. The decision to prescribe AIT is always tailor-made, balancing risk vs benefit. Creating globally accepted guidelines would help Allergologists in their decision making.
\end{abstract}

Keywords: Allergen immunotherapy, Venom hypersensitivity, Contraindications, Beta-blocker, Asthma, Autoimmunity, Malignancy, ACE-inhibitor, Pregnancy

\section{Background}

Allergen immunotherapy (AIT) is an evidence-based efficacious treatment option for respiratory and venom allergy, however, there are some concomitant diseases and underlying conditions that emerge as safety limitations and lead to contraindications to AIT $[1,2]$. Several controversies exist on these contraindications, like whether they are justified or not and their distinction in 'absolute' or 'relative'. Furthermore contraindications are different for subcutaneous airborne AIT (SCIT), for sublingual AIT (SLIT) and for subcutaneous venom immunotherapy (VIT). As a result of these controversies, there are clinical, legal and ethical issues that often arise [1].

\footnotetext{
*Correspondence: pitsios.constantinos@ucy.ac.cy

${ }^{1}$ Medical School, University of Cyprus, P.O. Box 20537, 1678 Nicosia,

Cyprus

Full list of author information is available at the end of the article
}

Due to ethical and practical reasons, it is not always possible to perform clinical trials on AIT's contraindications. Most of the existing studies regarding this topic are observational case-series or case-reports and only few evidence-based information regarding contraindications to AIT exist [1]. The decision to use AIT in patients with a contraindicated condition or concomitant disease is often based on risk-benefit balance; AIT may be justified in individual cases that are expected to be benefited more than posed to potential risk.

Guidelines on AIT, describing contraindications, have been developed and published by international academies and national societies of allergology and clinical immunology and are mainly based on experts' opinion [1-17]. In the frame of an EAACI Task Force, a position paper on contraindications has been published and 
the recent EAACI's Guidelines on Immunotherapy are in accordance with it $[18,19]$.

The present review has been produced by the EAACI Task Force on "Contraindications to Immunotherapy" and aims to offer a global overview on the topic revealing the differences of the different guidelines and existing contradictions.

\section{Method}

\section{Search strategy}

Guidelines, consensuses and position papers pertaining to AIT (for airborne allergens and Hymenoptera venoms) were retrieved from electronic bibliographic databases (Pubmed, Cochrane library, Google scholar). Specific immunotherapy; allergen immunotherapy; venom immunotherapy; sublingual; subcutaneous; guidelines and contraindications have been the individual search words for this research. The composite search terms were (allergen OR venom) AND immunotherapy AND (guidelines OR contraindication).

In cases that the search resulted in multiple guidelines from a single Society/Academy, the most recent one was preferred. The web sites of national academies and/or societies registered in the WAO and EAACI databases have been searched for official guidelines on AIT (and contraindications), in order to confirm that the most recent ones have been retrieved, or to obtain unpublished ones [20].

Furthermore, officially appointed contact-persons/ webmasters were approached by email, when the access to the official websites was allowed to members only. Not all webmasters replied and consequently an effort to contact directly the National Committees was made. Articles in various languages have been retrieved and translated into English, with the help of the respective national societies.

\section{Inclusion/exclusion criteria}

Guidelines that have been prepared and published under official auspicies of Societies and/or Academies that are members of WAO and/or registered in the WAO and EAACI databases, were included. Contraindications to AIT were searched for, including SLIT, SCIT and VIT.

Contraindications reported in guidelines of other nonallergy Societies/Colleges were excluded. Same for guidelines of Regional societies, when National guidelines where published. Reviews reported as "experts' opinion", that were not specified as official guidelines of a Society/ Academy of allergology/immunology, were also excluded.

\section{Categorization of contraindications}

In order to provide a uniform list of the suggested contraindications, an effort was made to minimize the heterogeneity of the various terms regarding the same disease/condition, merging them to more generic ones. However, when particular mentions on well-specified diseases or conditions had been made, these were listed separately from the generic terms. The use of the terms 'absolute' and 'relative' was not always clarified and in some guidelines other descriptive words had been used to replace them. Two reviewers (MT, CP) made the distinction of these terms in each guideline. Any discrepancies were resolved through discussion and, if necessary, a third reviewer was consulted (PD).

\section{Data synthesis}

A table including all diseases/conditions that have been described as contraindications and the relative Societies/ Academies, was created by MT, reviewed by CP and PD (Table 1). In the case that specific guidelines on different types of AIT have been retrieved, they are mentioned separately in Table 1.

\section{Results}

Guidelines' retrieval

A total of 544 publications was the outcome of the online research. 51 National and 4 International Societies/ Academies were contacted. Twenty-eight suitable papers have been retrieved; some of them have been published in official journals of national societies, not included in the main medical search engines. The exclusion of older guidelines of Societies, keeping the renewed ones, reduced the suitable papers to 21 . Although published in the Official journal of CSACI by members of its Board and followed by allergists, Canadian guidelines were not included because they do not consist official ones (personal communication) [21]. The inclusion criteria reduced the number of papers on contraindications from 21 to 17 (Fig. 1).

\section{Contraindication terms' selection}

A variety of contraindications was found, as described in Table 1. An heterogeneous use of terms has been used to describe the grade of contraindication and it was tried to reduce them to "absolute" or "relative". The terms "special considerations", "temporary precautions" and "reviewable" have been replaced by "relative", while the term "of major importance" has been replaced by "absolute" $[2,5]$.

\section{Guidelines to contraindications; the big picture}

Most of the Academies/Societies do not include official guidelines regarding contraindications to AIT in their websites. Some of them have accepted and reproduce official guidelines of other societies/academies, e.g. SLAAI refers to the AAAAI/ACAAI/JCAAI and CMICA guidelines, while HSACI follows both AAAAI and 


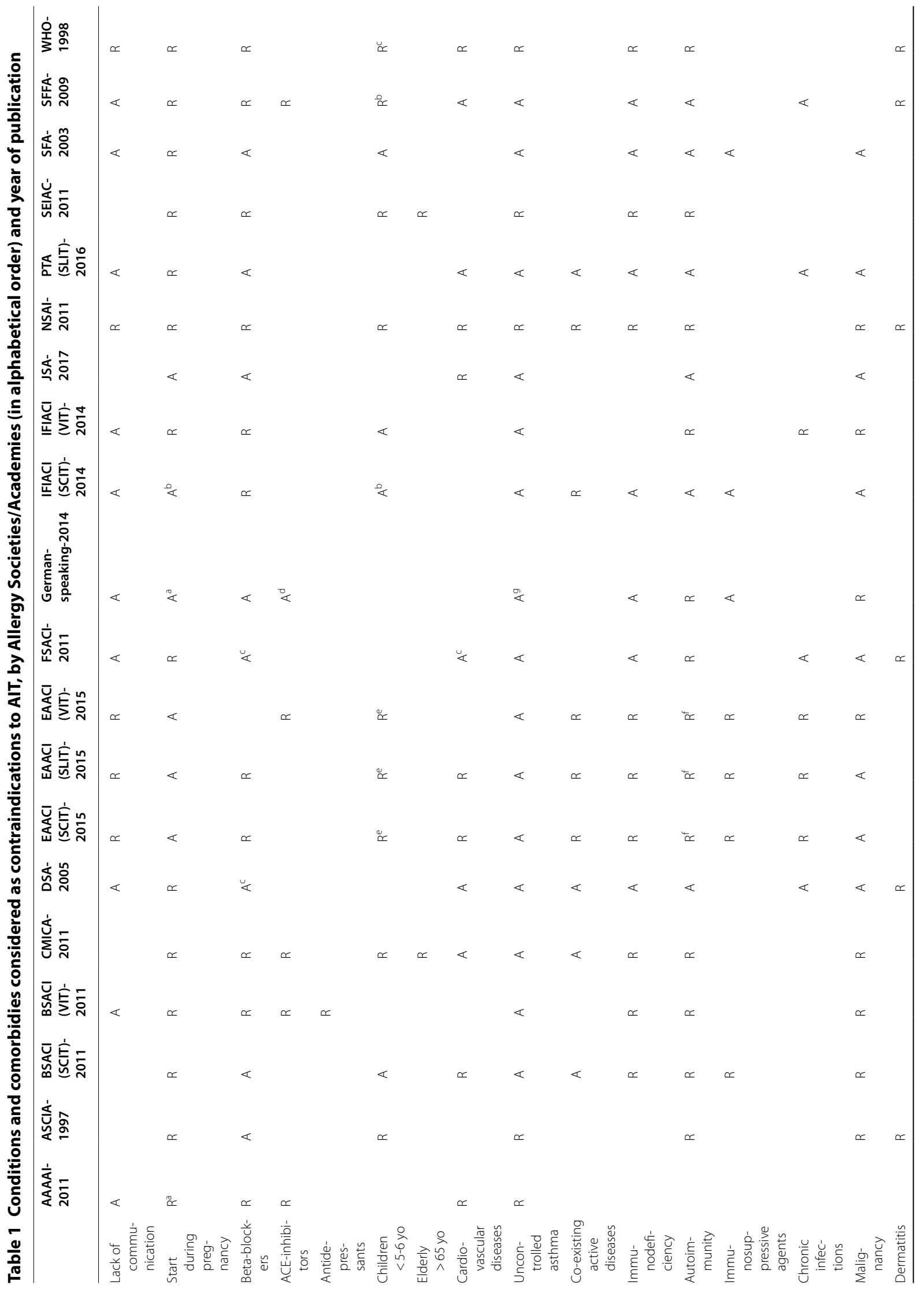




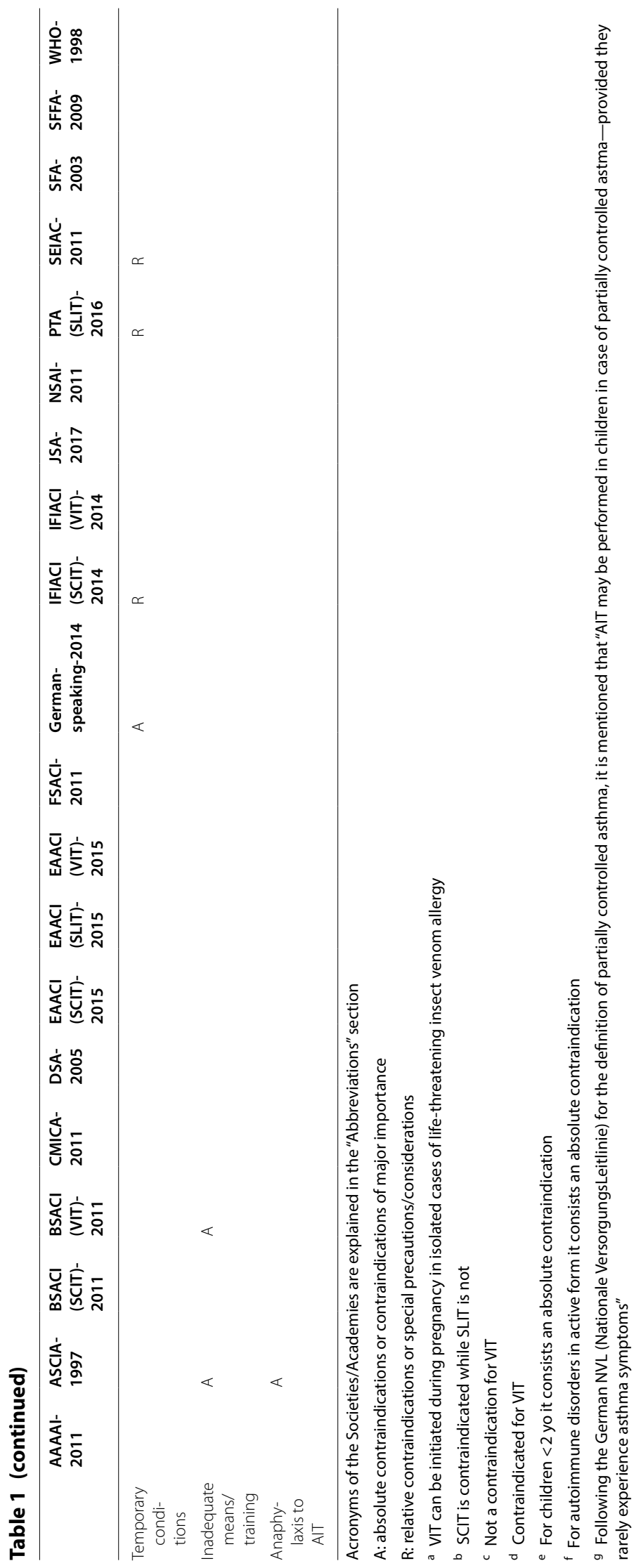




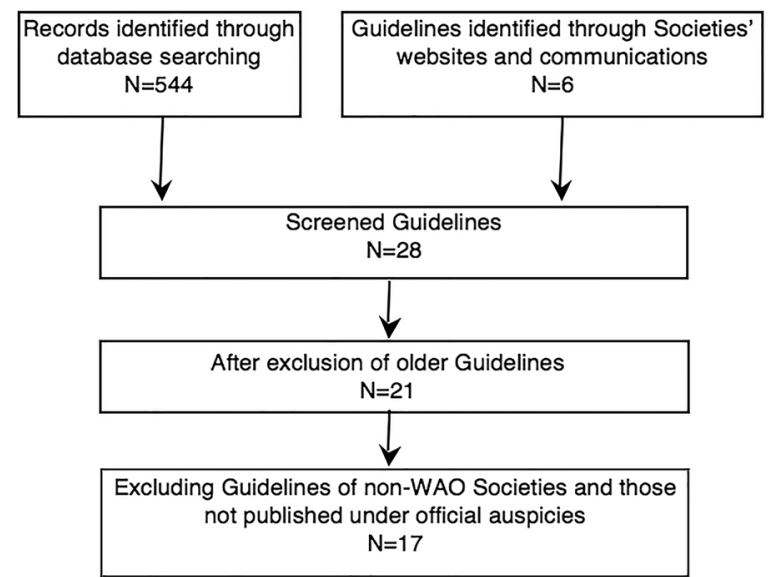

Fig. 1 Flow diagram of selection of guidelines on contraindications to AIT

EAACI ones. In some countries, like Argentina, there are different guidelines; AAAeIC uses WHO guidelines, while SAAeI uses CMICA and WHO guidelines (personal communication). Societies from German-speaking countries (Germany, Austria and Switzerland) have developed common guidelines [14].

Most guidelines refer to the administration of AIT with airborne allergens. In most Societies/Academies there are comments referring to the treatment of venom allergy and some contraindications don't apply as absolute for VIT. British and Italian Societies have developed special guidelines for Hymenoptera venom allergic patients [4, 22, 23].

Contraindications for SLIT are expressed in the position papers of German-speaking countries and of EAACI, while distinct guidelines have been developed in Poland by PTA $[1,14,17]$.

\section{Concomitant diseases and conditions described as contraindications}

A large heterogeneity among guidelines was noticed, however it is commonly accepted that: lack of communication (and/or cooperation), pregnancy before the start of AIT, treatment with beta-blockers, certain age groups, uncontrolled asthma, immune diseases and malignancies are included in most guidelines as (absolute or relative) contraindications to initiate AIT (Fig. 2) [1-17]. Inadequate medical means and AIT performed by clinicians without relative training are reported as absolute contraindications by ASCIA, also mentioning former anaphylaxis to AIT as a contraindication to continue [15]. Transient interfering situations (acute febrile illness, inflammatory and infective diseases, exacerbation of asthma) are mentioned by IFIACI under the term "temporary contraindications" [11]. Gastrointestinal inflammation, dental extraction or oral surgery and infections have been designated as temporal contraindications for SLIT $[8,14,17]$.

Initiation of AIT is contraindicated during pregnancy, while AIT can be continued if the woman became pregnant after starting it [1-17]. AAAAI guidelines for pregnancy mention that initiation of VIT might be considered in high-risk conditions, while in the case that a "patient is receiving a dose unlikely to be therapeutic, discontinuation of immunotherapy should be considered" [2]. Danish guidelines suggest to stop AIT when pregnancies' complications occur, or the patient expresses the slightest hesitation [9].

As far as drugs are concerned, cardiovascular drugs are of main consideration. In most guidelines it is suggested to replace beta-blockers (even when administered topically, e.g. eye drops) and/or ACE-inhibitors, with equally efficacious alternative drugs $[1,2,4,10,12,14]$. EAACI guidelines include beta-blockers as relative contraindication for SCIT and SLIT and ACE-inhibitors as relative ones for VIT [1]. BSACI guidelines mention the use of tricyclic antidepressants as a contraindication, suggesting their replacement by selective serotonin reuptake inhibitors, before commencement of VIT [4]. On the other hand the use of MAO-inhibitors, is currently very limited so the risk of their co-administration with epinephrine has elapsed.

Cardiovascular and lung diseases are comorbidities suggesting special considerations since they might result in impaired tolerance of hypotension, and bronchospasm, responding poorly to resuscitation and emergency treatment if AIT-induced anaphylaxis occurs [2-4, 7-10, $12-$ 14]. Asthma is highlighted in all guidelines and patients with severe asthma, with persistent symptoms remaining uncontrolled despite optimal pharmacological treatment and FEV $1<70 \%$ of the predicted value, are ruled out $[1-4,6-11,14]$. A convergence on severe/uncontrolled/ unstable asthma is noticed in the guidelines. German guidelines mention that AIT "may be performed in children in case of partially controlled asthma-provided they rarely experience asthma symptoms" [14].

Age limits were commented in most AIT guidelines recording infancy as a main concern (lower limit: 5 or 6 years of age in different guidelines) $[1-3,5-7,10,13]$. In CMICA guidelines, SCIT is indicated in children above 2 years old [7]. AIT has also been mentioned as a contraindication in elderly, because of high incidence of comorbid medical conditions and frequent use of the aforementioned cardiovascular drugs $[6,10]$.Concurrent depletion of the immune system in the cases of primary and secondary immunodeficiency syndromes, use of immunosuppressive agents, autoimmune diseases and 


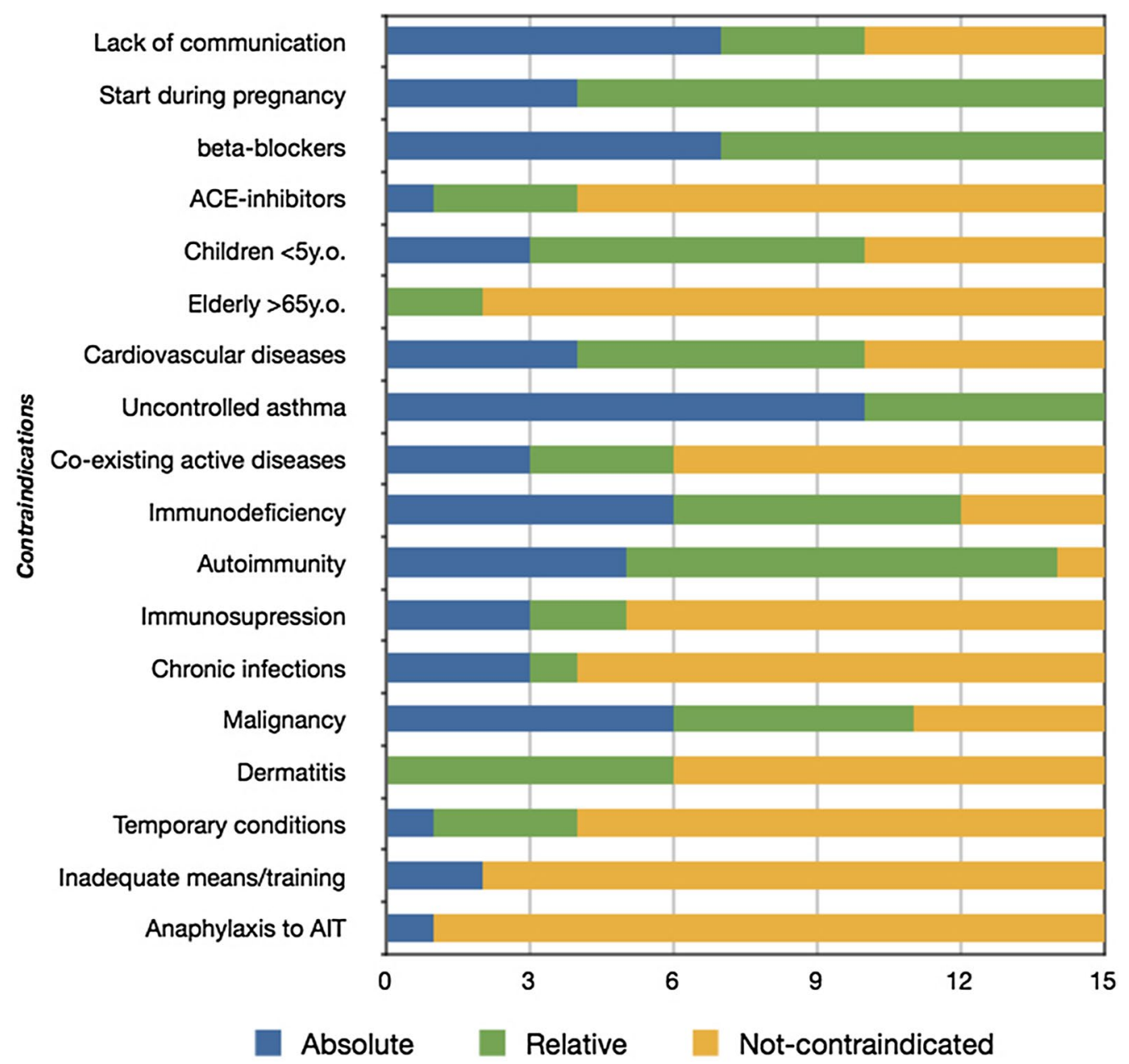

Fig. 2 Conflicting guidelines on whether a condition/comorbidity consists absolute, relative or no contraindication to SCIT

chronic infections are mentioned as contraindication in most guidelines, though there is no solid evidence that AIT is actually harmful in these patients [3-7, 9-14]. AIT is also contraindicated for patients with concomitant lymphoid malignancies and malignant tumors in general [3-7, 9-14]. In the article cited in SFA official website, a special reference appears suggesting to consider AIT in patients with healed tumors [5]. Regarding patients with HIV disease, AIT is contraindicated only at the AIDS stages $[1,2,19]$.

\section{Discussion}

Contraindications are developed in order to exclude patients with comorbid diseases to which AIT might constitute an aggravating co-factor, or who would not respond adequately to the treatment of anaphylaxis. Under some circumstances, an individual 'risk-benefit' analysis should be performed by specialists and AIT might be suggested even in high-risk patients ('relative contraindications'). As regards to the terms absolute and relative, their interpretation is often difficult, albeit they are useful to highlight that the decision to treat with AIT is not always a black and white issue. Hymenoptera venom allergy is potentially fatal, so VIT is considered a treatment option even when comorbidities exist.

There are data from retrospective (SCIT and VIT) and randomized controlled (SLIT) studies proving the safety of continuing AIT during pregnancy [24]. There are scarce data on the initiation of SCIT and SLIT during pregnancy reporting no maternal or fetal complications $[25,26]$, while initiation of AIT has also been reported by a limited number of allergists in CONSIT survey, without major problems [27]. Major problems were rarely noticed $(1.2 \%)$ by allergists who continued VIT in pregnant women [28]. In opinion surveys most responders would continue AIT (all types) during pregnancy [35, 36], would not start SCIT $[27,29]$ and don't consider the start of SLIT during pregnancy as a contraindication [27]. 
Based on old experimental studies (performed in humans and animals) and on surveillance surveys (regarding fatalities), the concomitant use of $\beta$-blockers is thought to constitute a risk factor for more severe and treatment-resistant anaphylaxis [30-34]. The use of ACE-inhibitors is also considered a risk-factor for serious hypotension in the case of allergic reactions during AIT, but caution is based on few case-reports [35]. Recently, with mouse model, it has been shown that the combined administration of $\beta$-blockers and ACE-inhibitors exacerbates anaphylactic symptoms, synergically increasing FceRI-mediated mastcell histamine release [36].

Real-life studies have provided data suggesting that $\beta$-blockers and ACE-inhibitors don't appear to increase the incidence of systemic reactions during VIT and SCIT [27, 35, 37-40]. SCIT has been associated with lower incidence of myocardial ischemia and infarction, compared with conventional therapy, so VIT is highly suggested in patients with cardiovascular diseases [41]. The presence of cardiovascular diseases in patients that are under $\beta$-blockers and ACE-inhibitors is a confounding factor for side-effects during AIT, so large prospective observational studies on the safety of these medicines, taking under consideration the underlying diseases, are needed [42].

Regarding lower age limits it has been suggested that AIT can be initiated in preschoolers (less than 5 years of age) if indications exist [1, 2, 7]. Performing SCIT and SLIT to toddlers has been proved to be safe and the prompt diagnosis of a systemic reaction in young children is easy to assess $[43,44]$. However, SCIT in infancy might be dangerous due to young children's inefficiency to communicate the symptoms pointing at the onset of anaphylaxis. Physicians have been discouraged from practising it in very young patients, due to limited published evidence supporting its benefit.

Although epidemiological studies support a decline of the prevalence of allergic diseases in elderly, there are immunological data suggesting that type-2 cytokines pattern is not defective in older age [45-47]. Even though debated, late-onset allergy to airborne allergens should be treated with AIT, taking under consideration concomitant diseases that pose contraindications. Benefits of AIT include protection when cardiovascular diseases coexist (as underlined in the case of VIT) [41], but also the reduced risk of side effects to the chronic use of corticosteroids (diabetes, osteoporosis, hypertension etc.) and anti-histamines (sedation and anti-cholinergic effect) [45].

Safety and effectiveness of AIT require adherence to the treatment and an adequate patient-physician collaboration. Psychiatric disorders are an heterogeneous group of mental health conditions. In the case that patients' ability to report symptoms suggestive of anaphylaxis has been impaired or a psychiatric condition that affects adherence to the treatment exists, AIT is contraindicated $[48,49]$. Collaboration with the caregiver and the psychiatrist, can increase adherence [50]. The heterogeneous symptomatology of psychiatric disorders should be taken into consideration and tailor-made decisions should be offered to patients. On the other hand non-adherence due to repeated forgetfulness or negligence (even in the absence of the aforementioned disorders) is a common problem in medicine, affecting safety and efficacy of AIT [51]. Continuing AIT is contraindicated to nonadherent patients, although no specific definitions in AIT guidelines have described which these limits are $[1,2,14]$.

There is a gap of evidence connecting AIT to an effect on autoimmune disorders. A paradox is the apparently beneficial use of honeybee stings for the treatment of rheumatoid arthritis, suggested by Acupuncture medicine [52], while VIT is contraindicated in patients with the same concomitant disease [1, 3-19]. The outcome of a large nationalwide study from Denmark, analyzing data over a decade, was that patients treated with AIT, receiving aluminium-containing allergen preparations, had lower incidence of autoimmunity compared to those on conventional treatment [41]. The evidence that AIT or the contained adjuvants can trigger or deteriorate autoimmune diseases in mainly based on a limited number of anecdotal case reports $[53,54]$. It would also be useful if guidelines were differentiating between practicing AIT with concomitant organ specific autoimmune disorders (Hashimoto thyroiditis, rheumatoid arthritis etc.) and systemic autoimmune disorders; German guidelines have done so [14].

The need to reassess neoplastic diseases for high-risk venom allergic patients, is also mentioned by guidelines [1, 11, 14]. Epidemiologic association between allergies and IgE levels (total and specific) with lower risk of developing certain malignancies has been expressed [55]. Although strong proof is missing, interfering with Th2 immunity may effect cancer; low dose ( 1 and $3 \mu \mathrm{g} / \mathrm{mL})$ of recombinant Der p 2 can enhance in vitro cell motility and invasiveness of non-small cell lung cancer cells, promoting metastatic ability of carcinoma cells [56]. On the contrary there are some data showing that upregulation of IgG4 antibodies offers protection from malignant melanocytes, so hypothetically their upregulation during AIT might benefit the cure of melanoma [57].

Defining contraindications to AIT is useful for allergists, though some of them require further clarification. As shown by CONSIT and by AAAAI's surveys, experienced allergists often use AIT beyond contraindications, on an individual basis [27-29]. CONSIT concluded that prescribing SLIT or performing VIT is less avoided 
when a relative contraindication occurs, and this is due to the high safety profile of SLIT or to the risk-benefit ratio favouring VIT [28]. However, AIT treatment in these cases should only be performed after thoroughly informing and training patients and their concordance is warranted.

There are ethical and legal conflicts on performing evidence-based clinical trials on patients with concomitant diseases. However, performing prospective surveillance studies by registering data of patients that are treated after giving informed consent can provide more concrete data. Such a study is currently contacted on the use of $\beta$-blockers and ACE-inhibitors, with preliminary results favouring treatment with AIT (personal communication). In the case of contraindications, the decision to prescribe AIT weighs risk vs benefit; decision is easier to be made in the case of VIT. The field of VIT contraindications can be investigated for most of relative contraindications and results may provide useful data that can apply to SLIT and SCIT. Regarding SLIT, since it is a safer alternative to SCIT, contraindications that apply may soon me minimized, given that long-term registration of case-series can be created.

\section{Conclusions}

A major heterogeneity regarding contraindications resulted from the present systematic review of the current literature in the field. Most guidelines are not evidence-based and reproduce older ones or copy each other. As AIT is evolving, novel forms of AIT are being produced and new data are arising, there is a profound need to update contraindications regularly. Ideally a globally accepted consensus on contraindications to AIT should be published aiming to reach international harmonization in this specific important domain of AIT. We believe that the present work paves the ground for such a future task.

\footnotetext{
Abbreviations

AAAAl: American Academy of Allergy Asthma and Immunology; AAAelC: Argentine Association of Allergy \& Clinical Immunology; ACAAl: American College of Allergy Asthma and Immunology; ÄDA: Association of German Allergists; ASCIA: Australasian society of Allergy and Clinical Immunology; BSACI: British Society for Allergy \& Clinical Immunology; CMICA: Mexican College of Clinical Immunology and Allergy; CSACl: Canadian Society of Allergy \& Clinical Immunology; DGAKI: German Society for Allergology and Clinical Immunology; DSA: Danish Society of Allergology; EAACl: European Academy of Allergy \& Clinical Immunology; FSACl: Finnish Society of Allergology and Clinical Immunology; GPA: Society of Pediatric Allergology and Environmental Medicine; HSACl: Hellenic Society of Allergology and Clinical Immunology; IFIACl: Federation of Italian Societies of Immunology, Allergology and Clinical Immunology; JCAAI: Joint Council of Allergy Asthma and Immunology; JSA: Japanese Society of Allergology; NSAI: Norwegian Society of Allergology and Immunopathology; ÖGAl: Austrian Society for Allergology and Immunology; PTA: Polish Society of Allergology; SAAel: Argentine Society of Allergy and Immunopathology; SEAIC: Spanish Society of Allergy and Clinical Immunology; SFA: French Society of Allergology; SFFA: Swedish Association for
}

Allergology; SLAAl: Latin American Society of Allergy, Asthma \& Immunology; SSAI/SGAI: Swiss Society of Allergology and Immunology; WAO: World Allergy Organization.

\section{Acknowledgements \\ None.}

\section{Authors' contributions}

This paper was drafted by CP, MT and the search strategy was developed by PD. It was initially revised following critical review by GS, OP, PRR and MBB, and then by all co-authors. This paper is part of the EAACI'Contraindications to Immunotherapy'TF, coordinated by CP. All authors read and approved the final manuscript.

\section{Funding}

EAACI.

Availability of data and materials

Not applicable.

Ethics approval and consent to participate

Not applicable.

\section{Consent for publication}

Not applicable.

\section{Competing interests}

C. Pitsios, M. Tsoumani, M.B. Bilò, R. Gawlik, F. Rueff, G. Paraskevopoulos, E. Valovirta and M.A. Calderon have no disclosures of interests to declare.O. Pfaar reports grants and personal fees from ALK-Abelló, grants and personal fees from Allergopharma, grants and personal fees from Stallergenes Greer, grants and personal fees from HAL Allergy Holding B.V./HAL Allergie GmbH, grants and personal fees from Bencard Allergie $\mathrm{GmbH}$ /Allergy Therapeutics, grants and personal fees from Lofarma, grants from Biomay, grants from Nuvo, grants from Circassia, grants and personal fees from ASIT Biotech Tools S.A. grants and personal fees from Laboratorios LETI/LETI Pharma, personal fees from Novartis Pharma, personal fees from MEDA Pharma, grants and personal fees from Anergis S.A., personal fees from Mobile Chamber Experts (a GA2LEN Partner), personal fees from Pohl-Boskamp, personal fees from Indoor Biotechnologies, grants from Glaxo Smith Kline, outside the submitted work.G. Sturm has received unrestricted research grant from ALK Abello, consulting and lecture fees from Novartis and Stallergens and lecture fees from Bencard, HAL and Allergopharma.P. Demoly has received personal fees for grants/lecture the past 2 years from AsraZeneca, Mylan, Sanofi, ASIT Biotech, ALK, StallergènesGreer, Thermofisher Scientific, Menarini, Chiesi, Bausch\&Lomb, Yslab.P. Rodruigez del Rio has received personal lecture fees from GSK, FAES, Novartis, ALK-Abelló, Merck, HAL Allergy Group, LETI and Aimmune Therapeutics.

\section{Author details}

${ }_{1}^{1}$ Medical School, University of Cyprus, P.O. Box 20537, 1678 Nicosia, Cyprus. 2 Division of Infection, Immunity and Respiratory Medicine, School of Biological Sciences, University of Manchester and NIHR Manchester Biomedical Research Centre, Manchester University Hospitals NHS Foundation Trust, Manchester Academic Health Science Centre, Manchester, UK. ${ }^{3}$ Dept. of Internal Medicine, Allergy Unit, University Hospital, Ancona, Italy. ${ }^{4}$ Dept. of Dermatology and Venereology, Medical University of Graz, Graz, Austria. ${ }^{5}$ Allergy Outpatient Clinic Reumannplatz, Vienna, Austria. ${ }^{6}$ Allergy Section, Children's University Hospital "Niño Jesús", Madrid, Spain. ${ }^{7}$ Dept. of Internal Medicine, Allergy and Clin. Immunology, Silesian University of Medicine, Katowice, Poland. ${ }^{8}$ Dermatology and Allergology Clinic and Policlinic, Ludwig-Maximilians University, Munich, Germany. ${ }^{9}$ Allergy Outpatient Clinic, 401 General Military Hospital of Athens, Athens, Greece. ${ }^{10}$ Terveystalo Turku, Allergy Clinic, University of Turku, Turku, Finland. ${ }^{11}$ Department of Otorhinolaryngology, Head and Neck Surgery, Universitätsmedizin Manneim, Medical Faculty Mannheim, Heidelberg University, Mannheim, Germany. ${ }^{12}$ Center for Rhinology and Allergology, Wiesbaden, Germany. ${ }^{13}$ Section of Allergy and Clinical Immunology, Imperial College London, National Heart and Lung Institute and Royal Brompton Hospital NSH, London, UK. ${ }^{14}$ Département de Pneumologie et Addictologie, Hôpital Arnaud de Villeneuve, University Hospital of Montpellier, Montpellier, France. ${ }^{15}$ UMR-S 1136, IPLESP, Equipe EPAR, Sorbonne Université, 75013 Paris, France. 
Received: 8 October 2018 Accepted: 4 September 2019

Published online: 11 September 2019

\section{References}

1. Pitsios C, Demoly P, Bilò MB, Gerth van Wijk R, Pfaar O, Sturm G, et al. Clinical contraindications to allergen immunotherapy: an EAACI position paper. Allergy. 2015;70:897-909.

2. Cox L, Nelson H, Lockey R, Calabria C, Chacko T, Finegold I, Nelson M, et al. Allergen immunotherapy: a practice parameter third update. J Allergy Clin Immunol. 2011;127(1 Suppl):S1-55.

3. Walker SM, Durham SR, Till SJ, Roberts G, Corrigan CJ, Leech SC, Krishna MT, et al. Immunotherapy for allergic rhinitis. Clin Exp Allergy. 2011:41:1177-200.

4. Krishna MT, Ewan PW, Diwakar L, Durham SR, Frew AJ, Leech SC, Nasser SM. Diagnosis and management of hymenoptera venom allergy: British Society for Allergy and Clinical Immunology (BSACl) guidelines. Clin Exp Allergy. 2011:41:1201-20.

5. Alvarez-Cuesta E, Beristain A. Conduite pratique de l'immunothérapie. Revue Fr Allergol. 2003;43:301-9.

6. De Luque-Piñana V, Guardia Martínez P, Moreno Aguilar C. Manejo práctico de inmunoterapia. In: Guardia Martínez P, Moreno Aguilar C, Tabar Purroy Al, editors. Inmunoterapia con alérgenos en el tratamiento de las enfermedades alérgicas, Comité de Inmunoterapia SEAIC-2010. Barcelona: ESMONpharma; 2011. p. 23-30.

7. Larenas-Linnemann D, Ortega-Martell J, del Río-Navarro B, RodríguezPérez N, Arias-Cruz A, Estrada A, Becerril-Ángeles M, et al. Guía Mexicana de Práctica Clínica de Inmunoterapia. Rev Alerg Mex. 2011;58:3-51.

8. Okubo K, Kurono Y, Ichimura K, Enomoto T, Okamoto Y, et al. Japanese Guideline for Allergic Rhinitis 2017. Allergol Int. 2017;66:205-19.

9. Lommeguide i praktisk allergen-specifik immunterapi. Baseret på EAAC Immunotherapy Interest Group Standards for Practical Allergen-Specific Immunotherapy. Danish Society for Allergology (Dansk Selskab for Allergologi, DSA), 2005.

10. Bolle R, Berstad A, Florvaag E, Steinsvåg S. Praktisk veileder I allergivaksinasjon 2. Norsk kvalitetssikringsdokument for Hyposensibilisering Allergenspesifikk immunterapi - Allergivaksinasjon. Norwegian Society of Allergology and Immunopathology (Norsk Forening for Allergologi og Immunpatologi, NSAI), 2011.

11. Musarra A, Bilò MB, Bonini S, Canonica GW, Senna GE. Consensus on allergen immunotherapy. Eur Ann Allergy Clin Immunol. 2014;45:S17-8.

12. Arvidsson $M$, Odebäck P, Eriksson M, Hammarlund A, Nilsson A, Sundler L, Söderberg M, Söderman P. Allergenspecifik immunterapi Rekommendationer för läkare och sjuksköterskor Revidering av tidigare rekommendationer från år 2000 Sammanställda på uppdrag av Svenska Föreningen för Allergologi 2009 Arbetet har finansierats av Svenska Föreningen För Allergologi. Arbetsgrupp Swedish Association For Allergology.

13. Bousquet J, Lockey R, Malling HJ. Allergen immunotherapy: therapeutic vaccines for allergic diseases A WHO position paper. J Allergy Clin Immunol. 1998;102:558-62.

14. Pfaar O, Bachert C, Bufe A, Buhl R, Ebner C, Eng P, Friedrichs F, Fuchs T, et al. Guideline on allergen-specific immunotherapy in lgE-mediated allergic diseases - S2k Guideline of the German Society for Allergology and Clinical Immunology (DGAKI), the Society for Pediatric Allergy and Environmental Medicine (GPA), the Medical Association of German Allergologists (AeDA), the Austrian Society for Allergy and Immunology (ÖGAI), the Swiss Society for Allergy and Immunology (SGAI), the German Society of Dermatology (DDG), the German Society of Oto-RhinoLaryngology, Head and Neck Surgery (DGHNO-KHC), the German Society of Pediatrics and Adolescent Medicine (DGKJ), the Society for Pediatric Pneumology (GPP), the German Respiratory Society (DGP), the German Association of ENT Surgeons (BV-HNO), the Professional Federation of Paediatricians and Youth Doctors (BVKJ), the Federal Association of Pulmonologists (BDP) and the German Dermatologists Association (BVDD). Allergo J Int. 2014;23:282-319.

15. Specific allergen immunotherapy for asthma. A position paper of the Thoracic Society of Australia and New Zealand and the Australasian Society of Clinical Immunology and Allergy. Med J Aust. 1997;167(10):540-4.

16. Valovirta E, Korhonen K, Kuitunen M, Kokkonen-Harjula K, Lammintausta K, Pallasaho P, et al. Allergen specific immunotherapy. Current care summary. Finnish Medical Society Duodecim and the Finnish Society of Allergology and Immunology; 2011.

17. Jutel M, Bartkowiak-Emeryk M, Breborowicz A, Cichocka-Jarosz E, Emeryk A, Gawlik R, Gonerko P, et al. Sublingual immunotherapy (SLIT)-indications, mechanism, and efficacy: position paper prepared by the Section of Immunotherapy, Polish Society of Allergy. Ann Agric Environ Med. 2016;23:44-53.

18. Roberts G, Pfaar O, Akdis CA, Ansotegui IJ, Durham SR, Gerth van Wijk R, Halken S, et al. EAACI Guidelines on allergen immunotherapy: allergic rhinoconjunctivitis. Allergy. 2017;73:765-98.

19. Sturm GJ, Varga EM, Roberts G, Mosbech H, Bilò MB, Akdis CA, AntolínAmérigo D, et al. EAACI Guidelines on allergen immunotherapy: hymenoptera venom allergy. Allergy. 2018;73:744-64.

20. http://www.worldallergy.org/about-wao/member-societies. Accessed 22 June 2018.

21. Moote W, Kim H. Allergen-specific immunotherapy. Allergy Asthma Clin Immmunol. 2011;7:S5

22. Linee Guida AAITO sulla Diagnosi e Terapia della Allergia al Veleno di Imenotteri. Editori: F.Bonifazi, M.B.Bilò. Con la collaborazione del Consiglio Direttivo AAITO: S.Amoroso, A.Antico, S.Ardito, G.Cadario, V.Feliziani, M.Galimberti, C.Lombardi, A.Musarra, G.Senna, G.F.Stefanini, C.Troise. Eur Ann Allergy Clin Immunol, 2004; S1: 1-24.

23. Pucci S, Arsieni A, Biale C, Ciccarelli A, Incorvaia C, Parzanese I, Passaleva G, et al. Linee Guida SIAIC sulla Diagnosi e Terapia della Allergia al Veleno di Imenotteri. Italian J Allergy Clin Immunol. 2005;15:139-61.

24. Oykhman P, Kim HL, Ellis AK. Allergen immunotherapy in pregnancy. Allergy Asthma Clin Immunol. 2015:11:31.

25. Shaikh WA. A retrospective study on the safety of immunotherapy in pregnancy. Clin Exp Allergy. 1993;23:857-60.

26. Shaikh WA. A prospective study on the safety of sublingual immunotherapy in pregnancy. Allergy. 2012;67:741-3.

27. Rodriguez Del Rio P, Pitsios C, Tsoumani M, Pfaar O, Paraskevopoulos G, Gawlik R, et al. Physicians' experience and opinion on contraindications to allergen immunotherapy. Ann Allergy Asthma Immunol. 2017;118:621-8.

28. Calabria CW, Hauswirth DW, Rank M, Sher L, Larenas-Linnemann D. American Academy of Asthma, Allergy \& Immunology membership experience with venom immunotherapy in chronic conditions and pregnancy, and in young children. Allergy Asthma Proc. 2017;38:121-9.

29. Larenas-Linnemann DE, Hauswirth DW, Calabria CW, Sher LD, Rank MA. American academy of allergy, asthma \& immunology membership experience with allergen immunotherapy safety in patients with specific medical conditions. Allergy Asthma Proc. 2016:37:112-22.

30. Hiatt WR, Wolfel EE, Stoll S, Nies AS, Zerbe GO, Brammell HL, et al. Beta-2 adrenergic blockade evaluated with epinephrine after placebo, atenolol, and nadiolol. Clin Pharmacol Ther. 1985;37:2-6.

31. Matsumura Y, Tan EM, Vaughan JH. Hypersensitivity to histamine and systemic anaphylaxis in mice with pharmacologic beta adrenergic blockade: protection by nucleotides. J Allergy Clin Immunol. 1976;58:387-94.

32. Nisam MR, Zbinden A, Chesrown S, Barnett D, Gold WM. Distribution and pharmacological release of histamine in canine lung in vivo. J Appl Physiol. 1978:44:455-63.

33. Kannan JA, Epstein TG. Immunotherapy safety: what have we learned from surveillance surveys? Curr Allergy Asthma Rep. 2013;13:381-8.

34. Reid MJ, Lockey RF, Turkeltaub PC, Platts-Mills TA. Survey of fatalities from skin testing and immunotherapy 1985-1989. J Allergy Clin Immunol. 1993;92:6-15.

35. Stoevesandt J, Hain J, Stolze I, Kerstan A, Trautmann A. Angiotensinconverting enzyme inhibitors do not impair the safety of Hymenoptera venom immunotherapy build-up phase. Clin Exp Allergy. 2014;44:747-55.

36. Nassiri M, Babina M, Dölle S, Edenharter G, Ruëff F, Worm M. Ramipril and metoprolol intake aggravate human and murine anaphylaxis: evidence for direct mast cell priming. J Allergy Clin Immunol. 2015;135:491-9.

37. Slade CA, Douglass JA. Changing practice: no need to stop ACE inhibition for venom immunotherapy. Clin Exp Allergy. 2014:44:617-9.

38. Muller UR, Haeberti G. Use of beta-blockers during immunotherapy for Hymenoptera venom allergy. J Allergy Clin Immunol. 2005:115:606-10.

39. Rueff F, Przybilla B, Bilo MB, Muller U, Scheipl F, Aberer W, et al. Predictors of side effects during the built-up phase of venom immunotherapy for Hymenoptera venom allergy: the importance of baseline serum tryptase. J Allergy Clin Immunol. 2010;126:105-15. 
40. Hepner MJ, Ownby DR, Anderson JA, Rowe MS, Sears-Ewald D, Brown EB. Risk of systemic reactions in patients taking beta-blocker drugs receiving allergen immunotherapy injections. J Allergy Clin Immunol. 1990;86:407-11

41. Linneberg A, Jacobsen RK, Jespersen L, Abildstrom SJ. Association of subcutaneous allergen-specific immunotherapy with incidence of autoimmune disease, ischemic heart disease, and mortality. J Allergy Clin Immunol. 2012;129:413-9.

42. Tejedor-Alonso MA, Farias-Aquino E, Rerez-Fernandez E, Grifol-Clar E, Moro-Moro M, Rosado-Ingelmo A. Relationship between anaphylaxis and use of beta-blockers and angiotensin-converting enzyme inhibitors: a systematic review and meta-analysis of observational studies. J Allery Clin Immunol Pract. 2018. https://doi.org/10.1016/j.jaip.2018.10.042.

43. Rodriguez-Perez N, Ambriz-Moreno MJ. Safety of immunotherapy and skin tests with allergens in children younger than five years. Rev Alerg Mex. 2006;53:47-51

44. Fiocchi A, et al. Safety of sublingual-swallow immunotherapy in children aged 3 to 7 years. Ann All Asthma Immunol. 2005;95:254-8.

45. Ridolo E, Rogkakou A, Ventoura MT, Martignano I, Incorvaia C, Di Lorenzo G, Passalaqua G. How to fit allergen immunotherapy in the elderly. Clin Mol Allergy. 2017;15:17.

46. Di Lorenzo G, Pacor ML, Esposito Pellitteri M, Listì F, Colombo A, Candore G, Mansueto P, Lo Bianco C, Ditta V, Battista Rini G, Caruso C. A study of age-related lgE pathophysiological changes. Mech Ageing Dev. 2003;124:445-8

47. Moro-García MA, Alonso-Arias R, López-Larrea C. Molecular mechanisms involved in the aging of the T-cell immune response. Curr Genomics. 2012;13:589-602.

48. Keith SJ, Kane JM. Partial compliance and patient consequences in schizophrenia: our patients can do better. J Clin Psychiatry. 2003;64:1308-15.

49. Piette JD, Heisler M, Ganoczy D, McCarthy JF, Valenstein M. Differential medication adherence among patients with schizophrenia and comorbid diabetes and hypertension. Psychiatric Serv. 2007;58:207-12.
50. Farooq S, Naeem F. Tackling nonadherence in psychiatric disorders: current opinion. Neuropsychiatr Dis Treat. 2014;10:1069-77.

51. Pitsios C, Dietis N. Ways to increase adherence to allergen immunotherapy. Curr Med Res Opin. 2018;23:1-9.

52. Lee JA, Son MJ, Choi J, Yun KJ, Jun JH, Lee MS. Bee venom acupuncture for rheumatoid arthritis: a systematic review protocol. BMJ Open. 2014;4:e004602.

53. Van der Laan JW, Gould S, Tanir JY, ILSI HESI Vaccines and Adjuvants Safety Project Committee. Safety of vaccine adjuvants: focus on autoimmunity. Vaccine. 2015;33:1507-14.

54. Ameratunga R, Gillis D, Gold M, Linneberg A, Elwood JM. Evidence refuting the existence of Autoimmune/Autoinflammatory Syndrome Induced by Adjuvants (ASIA). J Allergy Clin Immunol Pract. 2017;5:1551-5.

55. Jensen-Jarolim E, Bax HJ, Bianchini R, Capron M, Corrigan C, Castells M, et al. AllergoOncology - the impact of allergy in oncology: EAACI position paper. Allergy. 2017;72:866-87.

56. Lin $\mathrm{CH}$, Lin HH, Kuo CY, Kao SH. Aeroallergen Der $\mathrm{p} 2$ promotes motility of human non-small cell lung cancer cells via toll-like receptor-mediated up-regulation of urokinase-type plasminogen activator and integrin/focal adhesion kinase signaling. Oncotarget. 2017;14:11316-28.

57. Trampert DC, Hubers LM, van de Graaf SFJ, Beuers U. On the role of IgG4 in inflammatory conditions: lessons for lgG4-related disease. Biochim Biophys Acta Mol Basis Dis. 2018;1864:1401-9.

\section{Publisher's Note}

Springer Nature remains neutral with regard to jurisdictional claims in published maps and institutional affiliations.
Ready to submit your research? Choose BMC and benefit from:

- fast, convenient online submission

- thorough peer review by experienced researchers in your field

- rapid publication on acceptance

- support for research data, including large and complex data types

- gold Open Access which fosters wider collaboration and increased citations

- maximum visibility for your research: over $100 \mathrm{M}$ website views per year

At BMC, research is always in progress.

Learn more biomedcentral.com/submissions 